\title{
Potencial de Utilização de Cobertura Vegetal de Sorgo e Milheto na Supressão de Plantas Daninhas em Condição de CAMPO: II - E FeITOS DA Cobertura MORTA ${ }^{1}$
}

\author{
Potential of Sorghum and Pearl Millet Cover Crops in Weed Supression in the Field: II- \\ Mulching Effect
}

TREZZI, M.M. ${ }^{2}$ e VIDAL, R.A. ${ }^{3}$

\begin{abstract}
RESUMO - A capacidade de supressão de plantas daninhas por culturas de cobertura é bastante conhecida e explorada, embora seja pouco pesquisada a importância relativa dos efeitos fisicos e alelopáticos sobre esse fenômeno. Dois experimentos foram realizados a campo, em 1999/2000 e 2000/2001, na área experimental da Faculdade de Agronomia da UFRGS, no delineamento experimental em blocos ao acaso, com quatro repetições, objetivando determinar os efeitos da cobertura morta de plantas de sorgo e de milheto sobre a supressão de plantas daninhas. Nos dois anos de condução dos experimentos, os tratamentos resultaram de um fatorial, em que o fator A foi constituído pelos genótipos de sorgo RS 11, BR 601 e BR 304, representantes de três classes de produção de extratos radiculares hidrofóbicos em laboratório, pelo genótipo de milheto Comum RS e por uma testemunha sem culturas; e o fator B, constituído por niveis de palha de cada genótipo sobre o solo. Em 1999/2000, níveis de palha de sorgo de $1,3 \mathrm{t} \mathrm{ha}^{-1}$ foram suficientes para reduzir $50 \%$ das infestações de Brachiaria plantaginea (BRAPL) e Sida rhombifolia (SIDRH). Em 2000/2001, $4 \mathrm{t} \mathrm{ha}^{-1}$ de palha de sorgo ou milheto foram suficientes para reduzir 91, 96 e 59\% da população total de SIDRH, BRAPL e Bidens pilosa, respectivamente. A presença de resíduos da parte aérea de sorgo é mais importante na supressão de plantas daninhas do que a presença de resíduos das raízes dessa cultura.
\end{abstract}

Palavras-chave: Sorghum spp., Pennisetum americanum, níveis de palha, alelopatia, fatores físicos.

\begin{abstract}
The weed supression capacity of cover crops is well known and explored, although only a few works have been conducted on the relative importance of the physical and allelopathic effects on this phenomenon. Two trials were carried out in the field, in 1999/2000 and 2000/ 2001, at the experimental area of the University of Rio Grande do Sul, Brazil, arranged in a randomized experimental block design with four replications to evaluate the effects of sorghum and pearl millet mulch on weedsupression. In both experimental years, the treatments resulted in a factorial, where factor A was composed by sorghum genotypes RS 11, BR 601, and BR 304, representatives of three classes of hydrophobic root extract production in the laboratory; the pearl millet genotype Comum RS; and by a check without cultures. Factor B was formed by different levels of straw of each genotype over the soil. In 1999/2000, sorghum straw levels of 1.3 tha $\mathrm{h}^{1}$ were sufficient to decrease Brachiaria plantaginea (BRAPL) and Sida rhombifolia

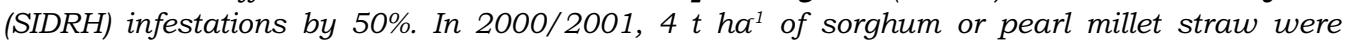
sufficient to decrease 91, 96, and 59\% of the total SIDRH, BRAPL, and Bidens pilosa population, respectively. The presence of sorghum shoot residues is more important in weed supression than the presence of root residues of this crop.
\end{abstract}

Key words: Sorghum spp., Pennisetum americanum, straw levels, allelopathy, physical factors.

Recebido para publicação em 29.10.2003 e na forma revisada em 5.3.2004.

Eng.-Agr., Dr., Professor Adjunto, Curso de Agronomia do CEFET/PR, Unidade de Pato Branco, Via do Conhecimento, Km 01, Caixa Postal 571, 95503-390 Pato Branco-PR. ${ }^{3}$ Eng.-Agr., Ph.D., Professor Adjunto, Faculdade de Agronomia da Universidade Federal do Rio Grande do Sul - UFRGS. 


\section{INTRODUÇÃO}

A supressão de plantas daninhas por coberturas mortas é atribuída a fatores de natureza física, química e biológica. A capacidade supressora de plantas daninhas por culturas de cobertura é amplamente reconhecida e explorada (Putnam et al., 1983; Almeida, 1988; Einhellig \& Rasmussen, 1989; Teasdale \& Mohler, 1993; Vidal \& Bauman, 1996; Theisen et al., 2000), embora não se conheça precisamente a importância relativa dos efeitos físicos e alelopáticos sobre esse fenômeno.

Em comparação ao solo desnudo, no mínimo $6 \mathrm{t} \mathrm{ha}^{-1}$ de palha de trigo foram necessários para reduzir em $50 \%$ a infestação de Setaria faberi (Vidal \& Bauman, 1996). Quantidades de palha de Avena strigosa de $10 \mathrm{t} \mathrm{ha}^{-1}$ não foram suficientes para impedir totalmente o estabelecimento de plantas de Brachiaria plantaginea (BRAPL) em soja (Theisen et al., 2000). A palha de sorgo é amplamente utilizada como cobertura por agricultores norte-americanos, objetivando a supressão de plantas daninhas (Putnam et al., 1983; Einhellig \& Rasmussen, 1989). Além de as plantas de sorgo possuírem capacidade de produção de níveis de palha mais elevados, é possivel que quantidades menores de palha dessa espécie sejam suficientes para resultar em supressão superior do crescimento de plantas daninhas, em relação aos resultados obtidos com resíduos de cereais de inverno.

Os efeitos físicos gerados pela palha estão relacionados principalmente à redução da disponibilidade da radiação solar (Facelli \& Pickett, 1991; Vidal, 1995) e à redução da amplitude térmica na camada superficial do solo (Teasdale \& Mohler, 1993; Vidal, 1995). Além dos efeitos físicos, compostos alelopáticos com capacidade de suprimir o crescimento de plantas daninhas podem ser liberados da cobertura morta de plantas cultivadas. A atividade alelopática de plantas de sorgo pode estar relacionada a compostos de natureza tanto hidrofílica quanto hidrofóbica.

Resíduos de sorgo em decomposição liberam compostos de natureza hidrofílica, em sua maioria ácidos fenólicos, como os ácidos ferúlico, vanílico, siríngico, p-hidroxibenzóico e, especialmente, p-cumárico (Guenzi \& McCalla,
1966; Nicollier et al. 1983; Weston et al., 1999). Variações consideráveis desses compostos foram encontradas em tecidos vegetais e no solo quando foram comparados três genótipos de sorgo (Ben-Hammouda et al., 1995).

Raízes de sorgo liberam compostos de natureza hidrofóbica, especialmente a benzoquinona sorgoleone, potente inibidor da respiração mitocondrial (Rasmussen et al., 1992) e, principalmente, do transporte de elétrons no fotossistema II (Einhellig et al., 1993; Nimbal et al., 1996; Gonzalez et al., 1997). Sorgoleone é capaz de suprimir diferencialmente o crescimento de várias espécies daninhas e cultivadas (Netzly et al., 1988; Einhellig \& Souza, 1992; Nimbal et al., 1996).

Foi observada pequena variação na produção de sorgoleone em coleções de 12 (Hess et al., 1992) e 10 (Rodrigues et al., 2001) genótipos de sorgo, embora Hess et al. (1992) tenham indicado que a produção variou segundo condições de ambiente. No entanto, obtevese variação considerável nas quantidades de sorgoleone produzidas, respectivamente, por 25 (Nimbal et al., 1996) e 41 (Trezzi, 2002) genótipos de sorgo.

O objetivo deste trabalho foi determinar os efeitos da cobertura morta de genótipos de sorgo com capacidade distinta de produção de extratos radiculares hidrofóbicos sobre a supressão de plantas daninhas, em campo.

\section{MATERIAL E MÉTODOS}

Foram realizados dois experimentos no campo, na Estação Experimental Agronômica (EEA) da Universidade Federal do Rio Grande do Sul (UFRGS), nos períodos de 1999/2000 e 2000/2001. O solo do local pertence à unidade de mapeamento São Jerônimo, sendo classificado como areno-argiloso (Argissolo Vermelho Distrófico típico) (Streck et al., 1999). As principais características do solo utilizado encontram-se na Tabela 1 .

As semeaduras dos genótipos de sorgo foram realizadas em 16/11/99 e 31/10/2000; no primeiro experimento, sobre cobertura de plantas de ervilhaca, e no segundo, sobre cobertura de plantas daninhas que se desenvolveram no local, ambas dessecadas previamente com $720 \mathrm{~g} \mathrm{ha}^{-1}$ de glyphosate. Foram 
Tabela 1 - Composição química e textural do solo utilizado nos experimentos. EEA/UFRGS, Eldorado do Sul-RS, 1999/2000 e $2000 / 2001$

\begin{tabular}{|c|c|c|c|c|c|c|c|c|c|c|}
\hline M.O. ${ }^{1 /}$ & Argila & \multirow{2}{*}{$\begin{array}{c}\mathrm{pH} \\
\left(\mathrm{H}_{2} \mathrm{O}\right)\end{array}$} & $\mathrm{P}$ & $\mathrm{K}$ & $\mathrm{Al}$ & $\mathrm{H}+\mathrm{Al}$ & $\mathrm{Ca}$ & $\mathrm{Mg}$ & C.T.C. ${ }^{2 /}$ & S.B. ${ }^{3 /}$ \\
\hline \multicolumn{2}{|c|}{$\left(\mathrm{g} \mathrm{kg}^{-1}\right)$} & & $\left(\mathrm{mg} \mathrm{dm}^{-3}\right)$ & \multicolumn{7}{|c|}{$\left(\mathrm{cmol}_{\mathrm{c}} \mathrm{dm}^{-3}\right)$} \\
\hline 29 & 290 & 5,5 & 30 & 0,52 & 0,0 & 2,5 & 3,6 & 2,0 & 8,6 & 6,12 \\
\hline
\end{tabular}

1/ Matéria orgânica; ${ }^{2 /}$ capacidade de troca de cátions; $\mathrm{e}^{3 / 2}$ saturação de bases.

utilizadas sementes visando o estabelecimento de 1.200.000 plantas ha ${ }^{-1}$, para cada genótipo de sorgo, e de 4.000 .000 plantas ha ${ }^{-1}$ de milheto.

Aos 14 dias após a semeadura, em 2000/ 2001, efetuou-se desbaste das plantas de sorgo, estabelecendo-se população de 1.200.000 plantas ha ${ }^{-1}$ para todos os genótipos. Após 48 (1999/2000) ou 63 dias (2000/2001) da semeadura, as plantas de sorgo e milheto (estádios $\mathrm{V}_{7} \mathrm{e}_{6}$, respectivamente, em sorgo e milheto) foram dessecadas com 720 g i.a. ha ${ }^{-1}$ de glyphosate.

Utilizou-se o delineamento em blocos ao acaso, com quatro repetições. Os experimentos foram organizados em parcelas subsubdivididas, considerando-se como parcelas principais os genótipos de sorgo RS 11, BR 601 e BR 304, representantes, respectivamente, das classes inferior, intermediária e superior de produção do aleloquímico sorgoleone (Trezzi, 2002); o genótipo de milheto Comum RS; e uma testemunha sem culturas. Como subparcelas, foi testada a utilização ou não de herbicida graminicida + óleo mineral (sethoxydim $230 \mathrm{~g} \mathrm{ha}^{-1}+$ Assist 1,5 $\mathrm{L} \mathrm{ha}^{-1}$ ) para o controle de plantas daninhas gramíneas em pósemergência; e, como subsubparcelas, niveis de palha sobre o solo. Em 1999/2000, através da movimentação da palha, a partir das quantidades originalmente produzidas, quatro niveis de palha foram estabelecidos nas subsubparcelas: ausência de cobertura de partes aéreas, nível original produzido no tratamento (1,0 $\left.\mathrm{tha}^{-1}\right)$, metade $\left(0,5 \mathrm{tha}^{-1}\right)$ e o dobro do nivel original $\left(2,0 \mathrm{t} \mathrm{ha}^{-1}\right)$. Em 2000/2001 foram estabelecidos apenas dois niveis, por meio da movimentação dos resíduos: ausência de cobertura e $4 \mathrm{t} \mathrm{ha}^{-1}$. A utilização ou não de herbicida graminicida foi concebida em função da interferência predominante que papuã
(Brachiaria plantaginea) pode exercer sobre espécies dicotiledôneas, em áreas com infestações mistas de plantas daninhas. Cada unidade experimental (subsubparcela) consistiu de área de $4 \mathrm{~m}^{2}(2 \times 2 \mathrm{~m})$. A aplicação de graminicida em pós-emergência ocorreu 15 e 17 dias após a movimentação da palha (DAM), respectivamente, em 1999/2000 e 2000/2001.

Foram identificadas as espécies de plantas daninhas e determinou-se sua densidade em área de $0,175 \mathrm{~m}^{2}$, aos 22 e 50 DAM em 1999/ 2000 e aos 21 e 56 DAM em 2000/2001. Aos 50 e 56 DAM, respectivamente, no primeiro e segundo períodos experimentais, também se determinou a massa seca das plantas daninhas, por meio do corte da parte aérea destas plantas em área de $0,175 \mathrm{~m}^{2}$, seguindo-se a secagem em estufa a $65^{\circ} \mathrm{C}$ por 72 horas. Para comparação da densidade populacional e massa seca de plantas daninhas entre médias de tratamentos, utilizou-se o teste da diferença mínima significativa (DMS), após os dados serem submetidos à análise de variância. Quando apropriado, ajustaram-se regressões entre niveis de palha e variáveis dependentes. Em geral, para os fatores testados, considerouse o nível de significância mínimo de $\mathrm{F}$ a $5 \%$. Em razão da complexidade que envolve a análise das interações entre fatores, considerouse o nível de significância mínimo de F a $15 \%$.

\section{RESULTADOS E DISCUSSÃO}

No primeiro ano (1999/2000) foram obtidas as seguintes quantidades originais de palha sobre a superficie: sorgo BR $304\left(1,0 \mathrm{t} \mathrm{ha}^{-1}\right)$, sorgo BR 601 (1,0 t ha-1), sorgo RS 11 (0,6 t ha $\left.{ }^{1}\right)$ e milheto Comum RS $\left(0,8 \mathrm{t} \mathrm{ha}^{-1}\right)$. A partir do nivel original, foram obtidos os niveis zero, metade, uma vez e o dobro. Nessas condições, hipotetizou-se que, em níveis semelhantes de 
palha na superfície do solo, as variações em infestação de plantas daninhas seriam decorrentes de diferenças na produção de substâncias alelopáticas. Para testar essa hipótese, foram utilizadas duas abordagens: na primeira, comparou-se o efeito dos genótipos de sorgo e milheto na ausência de palha (mas na presença de sistema radicular sob a superficie do solo); e, na segunda, foram comparados os genótipos de sorgo BR 304 e BR 601, que produziram niveis equivalentes de palha na superfície do solo, quanto à capacidade em reduzir a infestação de plantas daninhas.

As espécies daninhas predominantes na área experimental foram BRAPL e SIDRH, embora também houvesse ocorrência esparsa de plantas de BIDSS. Na ausência de palha sobre a superficie do solo, não foram observadas diferenças nas densidades de SIDRH e BRAPL entre os genótipos de sorgo e milheto (Figura 1), em quaisquer das épocas avaliadas (22 e 50 DAM). A massa seca de SIDRH foi superior onde não houve competição desta espécie com plantas daninhas gramineas, em comparação ao tratamento com competição (Tabela 2). Em função da utilização ou não de herbicida graminicida, foi constatada significância do fator graminicida para a variável massa seca de Brachiaria plantaginea (BRAPL).

Da mesma forma, não se observaram diferenças nas densidades de BRAPL, SIDRH e total entre os genótipos de sorgo BR 304 e BR 601 , em quaisquer dos niveis de palha testados, em ambas as avaliações, com exceção da ocorrência de maior população total de plantas daninhas no genótipo BR 601 em relação ao BR 304, no tratamento com duas vezes a quantidade original de palha, aos 22 DAM (Tabela 3).

Aos 22 DAM, houve redução das densidades populacionais de BRAPL, SIDRH e total, em 26, 65 e 95 plantas $\mathrm{m}^{-2}$, respectivamente, para cada tonelada de palha presente na superficie do solo, nas médias dos genótipos BR 304 e BR 601 (Figura 2 e Tabela 4), em relação aos tratamentos sem resíduo na superficie. Aos 50 DAM, taxas aproximadas de redução foram obtidas em relação à avaliação em 22 DAM, correspondendo a 26, 71 e 100 plantas $\mathrm{m}^{-2}$ para cada tonelada de palha

Tabela 2 - Massa seca de plantas de Sida rhombifolia (SIDRH), em função da utilização ou não de herbicida graminicida, médias de três genótipos de sorgo e um de milheto, sem o uso de palha das culturas na superfície. UFRGS, Eldorado do Sul-RS, 1999/2000

\begin{tabular}{|c|c|}
\hline Uso de graminicida & Massa seca de SIDRH $\left(\mathrm{g} \mathrm{m}^{-2}\right)$ \\
\hline Sem & $40 \mathrm{~b}^{\frac{1}{}}$ \\
Com & $110 \mathrm{a}$ \\
\hline CV & 19,33 \\
\hline
\end{tabular}

Médias seguidas da mesma letra não diferem significativamente a $1 \%$ pelo teste DMS.

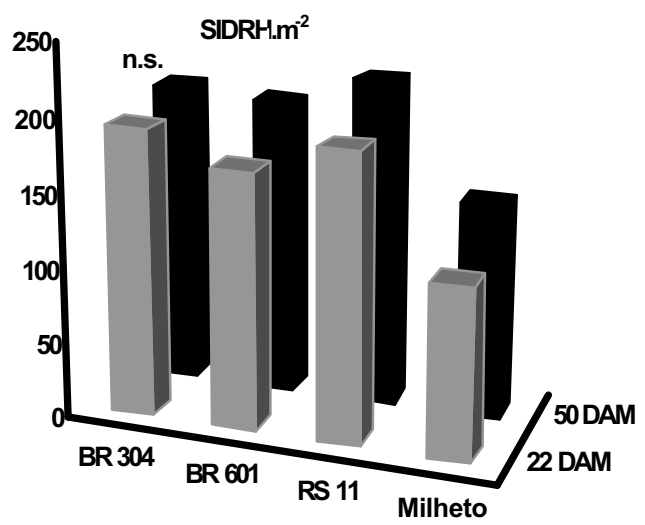

n.s. - comparação entre genótipos/espécie não-significativa a 5\% pelo teste $\mathrm{F}$.

Coeficientes de variação: BRAPL m ${ }^{2} 22$ DAM = 74,34\%; BRAPL m ${ }^{-2} 50$ DAM =71,68\%; SIDRH m $^{-2} 22$ DAM=34,54\%; SIDRH m ${ }^{-2} 50$ DAM $=34,28 \%$.

Figura 1 - Infestações de Brachiaria plantaginea (BRAPL) e Sida rhombifolia (SIDRH) emergidas em três genótipos de sorgo e um de milheto, na ausência de palha das culturas sobre a superfície do solo. UFRGS, Eldorado do Sul-RS, 1999/2000. 
adicionada, respectivamente para BRAPL, SIDRH e total (Figura 2 e Tabela 4).

Redução mais efetiva da densidade de plantas daninhas poderia ser obtida se a dessecação fosse realizada em estádios de desenvolvimento mais avançados $\left(\mathrm{V}_{6}\right.$ ou $\left.\mathrm{V}_{7}\right)$, obtendo-se, assim, maiores quantidades de palha na superficie do solo. Em comparação ao solo desnudo, no minimo $6 \mathrm{t} \mathrm{ha}^{-1}$ de palha de trigo foram necessários para reduzir em $50 \%$ a infestação

Tabela 3 - Densidade populacional total de plantas daninhas em resposta aos genótipos de sorgo BR 304 e BR 601 e quatro níveis de palha na superfície do solo, aos 22 e 50 dias após a movimentação da palha (DAM). UFRGS, Eldorado do Sul-RS, 1999/2000

\begin{tabular}{|l|c|c|c|c|}
\hline \multirow{2}{*}{ Genótipos } & \multicolumn{4}{|c|}{ Densidade total de plantas daninhas (plantas. ${ }^{-2}$ ) } \\
\cline { 2 - 5 } & $\begin{array}{c}\text { Sem palha } \\
\text { na superfície }\end{array}$ & $0,5 \mathrm{tha}^{-1}$ & $1,0 \mathrm{t} \mathrm{ha}^{-1}$ & $2,0 \mathrm{t} \mathrm{ha}^{-1}$ \\
\hline \multicolumn{5}{|c|}{ 22 DAM } \\
\hline BR 304 & a* 309 & a 147 & a 120 & b 33 \\
BR 601 & a 291 & a 140 & a 200 & a 136 \\
\hline \multicolumn{5}{|c|}{50 DAM } \\
\hline BR 304 & a 313 & a 136 & a 104 & a 35 \\
BR 601 & a 294 & a 168 & a 194 & a 135 \\
\hline
\end{tabular}

* Médias seguidas da mesma letra na coluna não diferem significativamente a $5 \%$ pelo teste DMS. Coeficientes de variação: densidade total de plantas daninhas $\mathrm{m}^{-2} 22 \mathrm{DAM}=45,77 \%$; densidade total de plantas daninhas $\mathrm{m}^{-2} 50 \mathrm{DAM}=41,31 \%$. de Setaria faberi (Vidal \& Bauman, 1996). No presente experimento, em geral, quantidades de palha de sorgo (médias dos genótipos BR 304 e BR 601 e de duas avaliações) de apenas 1,47 e $1,14 \mathrm{t} \mathrm{ha}^{-1}$ foram necessárias, respectivamente, para reduzir as infestações de BRAPL e SIDRH em 50\% (Tabela 4). No entanto, quantidade de palha de Avena strigosa de $10 \mathrm{t}$ $\mathrm{ha}^{-1}$ não foi suficiente para impedir totalmente o estabelecimento de plantas de BRAPL em soja (Theisen et al., 2000).

No segundo ano experimental (2000/2001) foram realizadas comparações entre todos os genótipos/espécies na ausência ou presença de $4 \mathrm{t} \mathrm{ha}^{-1}$ de palha na superficie do solo, considerando-se também a utilização ou não de herbicida graminicida.

A eliminação das plantas daninhas gramíneas pela utilização de graminicida não afetou a densidade de BIDSS, porém reduziu a população de plantas de SIDRH na primeira avaliação, aos 21 DAM (Tabela 5). Aos 56 DAM não se verificou este efeito, provavelmente em razão da mortalidade de plantas (autodesbaste) que se verifica sob populações elevadas (Radosevich et al., 1997). No entanto, a massa seca $\mathrm{m}^{-2}$ das plantas dessas espécies daninhas foi superior na ausência de competição com gramíneas, efeito observado apenas nos tratamentos sem cobertura morta na superficie
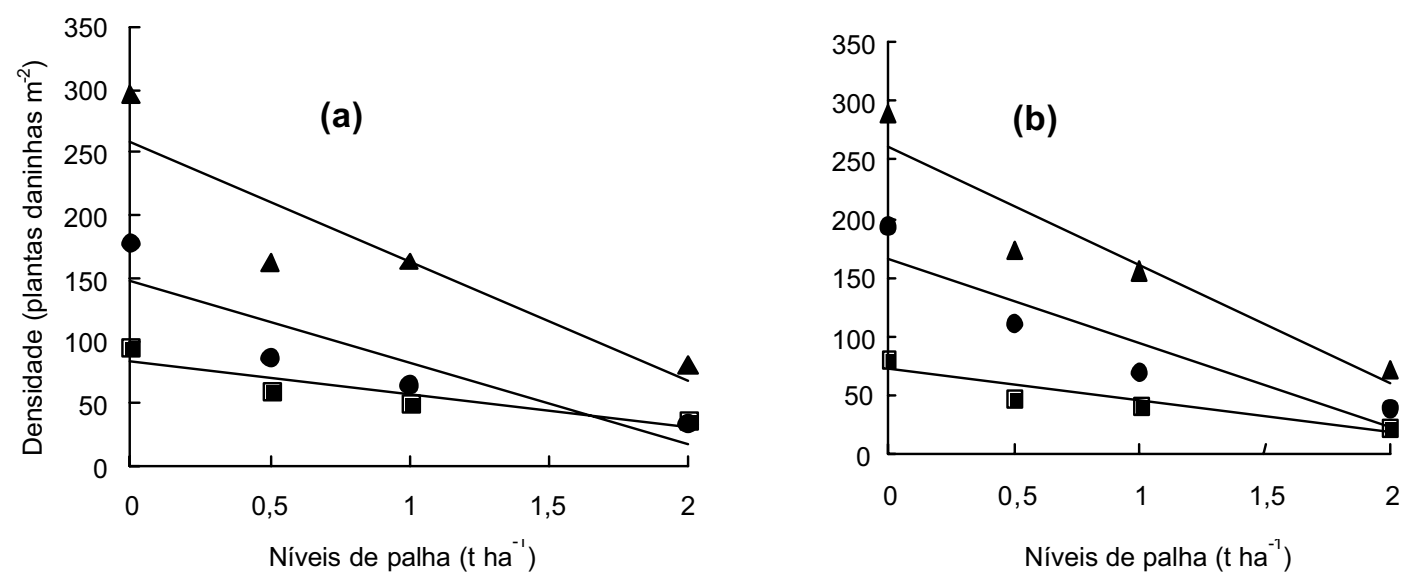

Equações de regressão linear, seus coeficientes de determinação e significância de F, encontram-se na Tabela 4.

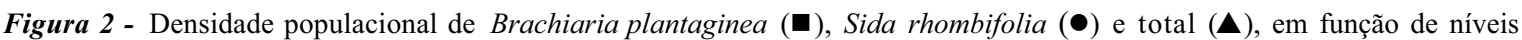
crescentes de palha sobre o solo, aos 22 (a) e 50 (b) dias após a sua movimentação (DAM), na média dos genótipos BR 304 e BR 601. UFRGS, Eldorado do Sul-RS, 1999/2000. 
do solo (Tabela 6). Isso pode ser explicado pelas densidades populacionais superiores de plantas daninhas em estádios precoces nos tratamentos sem cobertura morta e sem uso de herbicida graminicida (Tabela 5), em comparação aos tratamentos com presença de palha na superficie do solo.

A presença apenas das raízes em decomposição, dos cultivares de sorgo BR 601 e RS 11 e do cultivar de milheto Comum RS, incrementou a população de plantas daninhas aos 21 DAM, em comparação à testemunha sem as culturas (Tabela 7). Esse efeito não foi observado aos 56 DAM. As populações médias totais de plantas daninhas na testemunha sem a presença de cobertura foram de 604 e 483 plantas $\mathrm{m}^{-2}$, aos 21 e 56 DAM, respectivamente, enquanto na presença de raízes em decomposição elas foram de $1.217 \mathrm{e}$ 745 plantas $\mathrm{m}^{-2}$, respectivamente, na média dos genótipos (Tabela 7). Em experimento que testou o efeito de extratos radiculares de natureza hidrofilica, Trezzi (2002) verificou estimulação do crescimento da radícula e da parte aérea de plântulas de alface, mas não observou incremento na germinação final, nem houve aumento na velocidade de germinação desta espécie.

Tabela 4 - Equações, valores dos coeficientes de determinação e significância da regressão linear entre níveis de palha de sorgo e densidades de Brachiaria plantaginea (BRAPL), Sida rhombifolia (SIDRH) e total, aos 22 e 50 dias após a movimentação da palha (DAM). UFRGS, Eldorado do Sul-RS, 1999/2000

\begin{tabular}{|c|c|c|c|c|}
\hline Variável dependente & Época de avaliação & Equação & $r^{2}$ & $\mathrm{~F}^{1 /}$ \\
\hline \multirow{2}{*}{ Densidade de BRAPL } & 22 DAM & $y=83,0-26,11 x$ & 0,82 & $3,73^{2 /}$ \\
\hline & 50 DAM & $y=71,5-26,04 x$ & 0,87 & $6,54 *$ \\
\hline \multirow{2}{*}{ Densidade de SIDRH } & 22 DAM & $y=147,3-64,79 x$ & 0,80 & $7,25 *$ \\
\hline & 50 DAM & $y=165,7-71,60 x$ & 0,85 & $5,88 *$ \\
\hline \multirow{2}{*}{ Densidade total } & 22 DAM & $y=258,5-95,29 x$ & 0,83 & $5,22 *$ \\
\hline & 50 DAM & $\mathrm{y}=260,0-100,03 \mathrm{x}$ & 0,90 & $8,00 * *$ \\
\hline
\end{tabular}

* Significativo a $5 \% ; *$ significativo a $1 \% ;{ }^{1 /}$ valores originais transformados para $\log (\mathrm{x}+10) ; \mathrm{e}^{2 /}$ significativo a $6 \%$.

Tabela 5 - Densidades populacionais de Brachiaria plantaginea (BRAPL), Sida rhombifolia (SIDRH) e Bidens sp. (BIDSS) aos 21 e 56 dias após a movimentação da palha (DAM), em resposta à presença ou não de cobertura morta de sorgo/milheto e de espécies daninhas gramíneas. UFRGS, Eldorado do Sul-RS, 2000/2001

\begin{tabular}{|c|c|c|c|c|c|c|c|c|c|}
\hline \multirow{3}{*}{$\begin{array}{l}\text { Herbicida } \\
\text { graminicida }\end{array}$} & \multicolumn{9}{|c|}{ Densidade de plantas daninhas (plantas $\mathrm{m}^{-2}$ ) } \\
\hline & \multicolumn{3}{|c|}{ BRAPL } & \multicolumn{3}{|c|}{ SIDRH } & \multicolumn{3}{|c|}{ BIDSS } \\
\hline & Com palha & Sem palha & Médias & Com palha & Sem palha & Médias & Com palha & Sem palha & Médias \\
\hline \multicolumn{10}{|c|}{$21 \mathrm{DAM}$} \\
\hline Sem & $21 \mathrm{~B}^{*}$ & $579 \mathrm{~A}$ & 300 & 14 & 195 & $104 \mathrm{a}$ & a $39 \mathrm{~B}$ & a $96 \mathrm{~A}$ & 115 \\
\hline Com & - & - & - & 6 & 96 & $51 \mathrm{~b}$ & a $46 \mathrm{~A}$ & a $59 \mathrm{~A}$ & 52 \\
\hline Médias & - & - & & $10 \mathrm{~B}$ & $145 \mathrm{~A}$ & & 43 & 76 & \\
\hline \multicolumn{10}{|c|}{56 DAM } \\
\hline Sem & $22 \mathrm{~B}$ & $346 \mathrm{~A}$ & 184 & 16 & 115 & $65^{\text {n.s. }}$ & $35^{\text {n.s. }}$ & 38 & $36^{\text {n.s. }}$ \\
\hline Com & - & - & - & 19 & 104 & 61 & 44 & 45 & 44 \\
\hline Médias & - & - & & $18 \mathrm{~B}$ & $109 \mathrm{~A}$ & & $39^{\text {n.s. }}$ & 41 & \\
\hline
\end{tabular}

* Médias seguidas da mesma letra maiúscula na linha e minúscula na coluna não diferem significativamente a 5\% pelo teste DMS. n.s. - Não-significativo.

CV BRAPL m ${ }^{-2} 21$ DAM: subparcela $=19,7 \%$; subsubparcela $=15,3 \%$; CV BRAPL m ${ }^{-2} 56$ DAM: subparcela $=19,1 \%$; subsubparcela $=$ 19,1\%; CV SIDRH m 21 DAM: subparcela $=12,8 \%$; subsubparcela $=20,1 \%$; CV SIDRH $\mathrm{m}^{-2} 56$ DAM: subparcela $=16,9 \%$; subsubparcela $=20,6 \%$; CV BIDSS $\mathrm{m}^{-2} 21$ DAM: subparcela $=13,1 \%$; subsubparcela $=16,9 \%$; CV BIDSS $\mathrm{m}^{-2} 56$ DAM: subparcela $=$ $10,2 \%$; subsubparcela $=16,6 \%$.

Planta Daninha, Viçosa-MG, v.22, n.1, p.1-10, 2004 
Tabela 6 - Massa seca de Brachiaria plantaginea (BRAPL), Sida rhombifolia (SIDRH) e Bidens sp. (BIDSS), determinada aos 56 dias após a movimentação de palha (DAM), em função da presença ou não de palha de sorgo/milheto na superfície do solo e de espécies daninhas gramíneas. UFRGS, Eldorado do Sul-RS, 2000/2001

\begin{tabular}{|c|c|c|c|c|c|c|c|c|}
\hline \multirow{2}{*}{$\begin{array}{c}\text { Herbicida } \\
\text { graminicida }\end{array}$} & \multicolumn{9}{|c|}{ Massa seca de plantas daninhas $\left(\mathrm{g} \mathrm{m}^{-2}\right)$} \\
\cline { 2 - 9 } & \multicolumn{2}{|c|}{ BRAPL } & \multicolumn{3}{c|}{ SIDRH } & \multicolumn{3}{c|}{ BIDSS } \\
\cline { 2 - 9 } & Com palha & Sem palha & Com palha & Sem palha & Médias & Com palha & Sem palha & Médias \\
\hline Sem & $23 \mathrm{~B} *$ & $141 \mathrm{~A}$ & $\mathrm{a} 3 \mathrm{~A}$ & $\mathrm{~b} 10 \mathrm{~A}$ & 6 & a $29 \mathrm{~A}$ & $\mathrm{~b} 20 \mathrm{~A}$ & 24 \\
\hdashline Com & - & - & a $4 \mathrm{~B}$ & $\mathrm{a} 32 \mathrm{~A}$ & 18 & a $40 \mathrm{~A}$ & a $59 \mathrm{~A}$ & 49 \\
\hline Médias & - & - & 3 & 21 & & 34 & 39 & \\
\hline
\end{tabular}

* Médias seguidas da mesma letra maiúscula na linha e minúscula na coluna não diferem significativamente a 5\% pelo teste DMS.

$\mathrm{CV}$ massa seca BRAPL: subparcela $=22,5 \%$; subsubparcela $=16,1 \%$; $\mathrm{CV}$ massa seca $\mathrm{SIDRH}$ : subparcela $=12,3 \%$; subsubparcela $=$ 23,4\%; CV massa seca BIDSS: subparcela $=14,7 \%$; subsubparcela $=19,5 \%$.

Tabela 7 - Densidade total de plantas daninhas avaliada aos 21 e 56 dias após a movimentação de palha (DAM), em genótipos de sorgo e milheto, na ausência de herbicida graminicida e de palha na superfície do solo (mas na presença de raízes em decomposição), em comparação com testemunha sem presença de resíduos da parte aérea e de raízes das culturas. UFRGS, Eldorado do Sul-RS, 2000/2001

\begin{tabular}{|c|c|c|}
\hline \multirow[t]{2}{*}{ Tratamento } & \multicolumn{2}{|c|}{$\begin{array}{l}\text { Densidade total de plantas daninhas } \\
\text { (plantas } \mathrm{m}^{-2} \text { ) }\end{array}$} \\
\hline & $21 \mathrm{DAM}$ & 56 DAM \\
\hline Sorgo BR 304 & $1.078 \mathrm{ab}^{1 /}$ & $631^{\text {n.s. }}$ \\
\hline Sorgo BR 601 & $1.098 \mathrm{a}$ & 694 \\
\hline Sorgo RS 11 & $1.400 \mathrm{a}$ & 830 \\
\hline Milheto Comum RS & $1.293 \mathrm{a}$ & 824 \\
\hline Testemunha sem cultura & $604 \mathrm{~b}$ & 483 \\
\hline Valores de F (tratamentos) & $3,80^{*}$ & $1,34^{\text {n.s. }}$ \\
\hline CVparcela (\%) & 28,6 & 36,0 \\
\hline
\end{tabular}

${ }^{1 /}$ Médias seguidas da mesma letra na coluna não diferem signifi cativamente a $5 \%$ pelo teste DMS; n.s. - não-significativo; e * significativo a $5 \%$.

A presença de $4 \mathrm{t} \mathrm{ha}^{-1}$ de palha de sorgo ou milheto na superfície do solo reduziu a população de SIDRH em 93 e 83\%, aos 21 e 56 DAM, respectivamente, em relação à ausência de palha na superficie do solo (porém com presença das raizes das plantas) (Tabela 5). No tratamento em que não se utilizou herbicida graminicida, a presença de palha reduziu as densidades de BRAPL em 96 e 94\%, aos 21 e 56 DAM, respectivamente, e de BIDSS em 59\%, aos 21 DAM (Tabela 5). Sem uso de herbicida graminicida, a população total de plantas daninhas foi reduzida em 91 e $84 \%$, aos 21 e 56 DAM, respectivamente, em relação à ausência de palha na superficie do solo. Com a utilização de herbicida graminicida, as reduções da população total foram de 72 e $60 \%$, aos 21 e 56 DAM, respectivamente (Tabela 8).

Houve redução média de $62 \%$ na massa seca total de plantas daninhas pelo efeito da palha (Tabela 8), devido principalmente à redução de $84 \%$ da massa de BRAPL, no tratamento sem uso de herbicida graminicida, e de $87 \%$ da massa de SIDRH, no tratamento com utilização de graminicida (Tabela 6). Niveis de controle de Galinsoga parviflora e de Richardia brasiliensis de 92 e $83 \%$, respectivamente, com cobertura morta de sorgo obtida pela densidade de semeadura de 200.000 plantas ha-1, foram observados por Peixoto et al. (2000).

Na média das duas datas de avaliação, as coberturas de milheto e sorgo foram capazes de reduzir, respectivamente, em 75 e $80 \%$ a infestação de total de plantas daninhas, em comparação à ausência de cobertura do solo (Tabela 9). Nas duas datas de avaliação, nos tratamentos com cobertura de palha, houve menor densidade total de plantas daninhas no genótipo de sorgo BR 601 do que em RS 11 (Tabela 9). Também, constatou-se, nos tratamentos com palha, menor massa seca de BRAPL em BR 601 e BR 304, em relação ao cultivar Comum de milheto (Tabela 10). No entanto, não foram detectadas diferenças entre genótipos nos tratamentos sem cobertura de palha. O cultivar de sorgo BR 601 também reduziu com maior intensidade a população total de plantas daninhas nas linhas de semeadura, em relação aos demais genótipos de sorgo, na fase em que as plantas da cultura 
Tabela 8 - População e massa seca total de plantas daninhas avaliadas aos 21 e 56 dias após a movimentação da palha (DAM), em função da presença ou não de palha na superfície do solo e de espécies daninhas gramíneas. UFRGS, Eldorado do SulRS, 2000/2001

\begin{tabular}{|c|c|c|c|c|c|}
\hline \multirow{2}{*}{$\begin{array}{l}\text { Herbicida } \\
\text { graminicida }\end{array}$} & \multicolumn{2}{|c|}{$\begin{array}{c}\text { População total } \\
\text { (plantas } \mathrm{m}^{-2} \text { ) }\end{array}$} & \multicolumn{2}{|c|}{$\begin{array}{c}\text { Massa seca total } \\
\left(\mathrm{g} \mathrm{m}^{-2}\right)\end{array}$} & \\
\hline & Com palha & Sem palha & Com palha & Sem palha & \\
\hline \multicolumn{5}{|c|}{$21 \mathrm{DAM}$} & \\
\hline Sem & a 104 B* & a $1.115 \mathrm{~A}$ & $\therefore$ & $\therefore$ & \\
\hline Com & a $57 \mathrm{~A}$ & b $203 \mathrm{~B}$ & - & - & \\
\hline \multicolumn{5}{|c|}{56 DAM } & Média \\
\hline $\begin{array}{l}\text { Sem } \\
\text { Com }\end{array}$ & $\begin{array}{lr}\text { a } & 107 \mathrm{~B} \\
\text { a } & 81 \mathrm{~B} \\
\end{array}$ & $\begin{array}{l}\text { A } 682 \mathrm{~A} \\
\text { b } 201 \mathrm{~A}\end{array}$ & $\begin{array}{l}78 \\
50\end{array}$ & $\begin{array}{l}213 \\
116\end{array}$ & $\begin{array}{l}\text { b } 46 \\
\text { a } 83\end{array}$ \\
\hline Média & & & $63 \mathrm{~B}$ & $164 \mathrm{~A}$ & \\
\hline
\end{tabular}

* Médias seguidas da mesma letra maiúscula na linha e minúscula na coluna não diferem significativamente a 5\% pelo teste DMS.

$\mathrm{CV}$ massa seca total: subparcela $=10,4 \%$; subsubparcela $=11,8 \%$; CV população total 21 DAM: subparcela $=12,4 \%$; subsubparcela $=$ $14,4 \%$; CV população total 56 DAM: subparcela $=10,4 \%$; subsubparcela $=11,8 \%$.

Tabela 9 - Densidade total de plantas daninhas aos 21 e 56 dias após a movimentação da palha (DAM), em função da presença ou não de cobertura morta na superfície do solo e genótipos/espécies de plantas cultivadas. UFRGS, Eldorado do Sul-RS, $2000 / 2001$

\begin{tabular}{|c|c|c|c|c|}
\hline \multirow{3}{*}{ Palha } & \multicolumn{4}{|c|}{ Genótipo/Espécie } \\
\hline & Sorgo BR 304 & Sorgo BR 601 & Sorgo RS 11 & Milheto \\
\hline & \multicolumn{4}{|c|}{ Densidade total de plantas daninhas (plantas $\mathrm{m}^{-2}$ ) } \\
\hline \multicolumn{5}{|c|}{ Aos 21 DAM } \\
\hline $\begin{array}{l}\text { Com } \\
\text { Sem }\end{array}$ & $\begin{array}{c}\mathrm{b}^{*} 64 \mathrm{AB} \\
\text { a } 506 \mathrm{~A}\end{array}$ & $\begin{array}{l}\text { b } 42 \mathrm{~B} \\
\text { a } 533 \mathrm{~A}\end{array}$ & $\begin{array}{l}\text { b } 131 \mathrm{~A} \\
\mathrm{a} 407 \mathrm{~A}\end{array}$ & $\begin{array}{c}\text { b } 98 \mathrm{AB} \\
\text { a } 480 \mathrm{~A}\end{array}$ \\
\hline \multicolumn{5}{|c|}{ Aos 56 DAM } \\
\hline $\begin{array}{l}\text { Com } \\
\text { Sem }\end{array}$ & $\begin{array}{c}\text { b } 87 \mathrm{AB} \\
\text { a } 362 \mathrm{~A}\end{array}$ & $\begin{array}{l}\text { b } 57 \mathrm{~B} \\
\text { a } 407 \mathrm{~A}\end{array}$ & $\begin{array}{l}\text { b } 134 \mathrm{~A} \\
\text { a } 360 \mathrm{~A}\end{array}$ & $\begin{array}{c}\text { b } 113 \mathrm{AB} \\
\text { a } 361 \mathrm{~A}\end{array}$ \\
\hline
\end{tabular}

* Médias seguidas da mesma letra maiúscula na linha e minúscula na coluna dentro do fator genótipos/espécies ou níveis de palha não diferem significativamente a $5 \%$ pelo teste DMS.

$\mathrm{CV}$ população total 21 DAM: parc ela $=9,2 \%$; subsubparcela $=14,4 \%$; CV população total 56 DAM: parcela $=6,3 \%$; subsubparcela $=$ $11,8 \%$.

estavam vivas, em experimento conduzido em 2000/2001 (Trezzi, 2002). Esses resultados concordam, em parte, com os dados obtidos em laboratório, em que, na concentração mais discriminatória de extratos hidrofílicos da parte aérea $(1,17 \%)$, o cultivar de sorgo BR 601 foi mais tóxico que os demais (Trezzi, 2002). Contudo, os extratos da parte aérea de milheto foram mais tóxicos do que os dos genótipos de sorgo, ao contrário do que se verificou no presente experimento.

Comparando efeitos de diferentes partes de plantas de sorgo em um experimento em baldes, Hoffman et al. (1996) não observaram diferenças sobre a emergência de Echinochloa crusgalli e Abutilon theophrasti, porém verificaram redução mais pronunciada no crescimento das raízes do que da parte aérea das plantas.

Os resultados do presente trabalho indicam pequena probabilidade de exsudatos radiculares hidrofóbicos de sorgo exercerem efeito sobre a emergência e o desenvolvimento de plantas daninhas após a dessecação das plantas de sorgo. Sorgoleone, o principal componente dos extratos hidrofóbicos de sorgo, é 
Tabela 10 - Massa seca de Brachiaria plantaginea (BRAPL) e Sida rhombifolia (SIDRH), em função dos fatores genótipos/espécies, presença ou não de palha na superfície do solo e do uso de herbicida graminicida. UFRGS, Eldorado do Sul-RS, 2000/2001

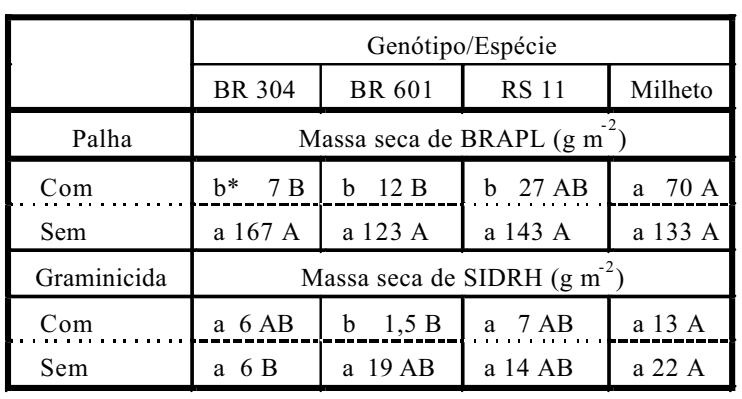

* Médias seguidas da mesma letra maiúscula na linha e minúscula na coluna dentro do fator genótipos/espécies ou herbicida graminicida não diferem significativamente a $5 \%$ pelo teste DMS.

CV massa seca BRAPL: parcela $=12,3 \%$; subsubparcela $=16,1 \%$. $\mathrm{CV}$ massa seca SIDRH: parcela $=11,4 \%$; subparcela $=12,3 \%$.

liberado das raízes para o solo (Netzly et al., 1988), porém, em razão de sua baixa mobilidade nesse meio, é provável que sua ação, caso exista alguma, restrinja-se à região próxima às raízes das plantas.

A presença de resíduos da parte aérea das plantas das culturas foi mais inibitória a plantas daninhas do que a presença de resíduos de raizes. A parte aérea das plantas pode mostrar maior atividade sobre plantas daninhas devido a efeitos físicos ou alelopáticos. A redução na disponibilidade de radiação solar (Facelli \& Pickett, 1991; Vidal, 1995) e na amplitude térmica na camada superficial do solo coberto com palha (Teasdale \& Mohler, 1993; Vidal, 1995) pode resultar em menores taxas de germinação e emergência de plantas daninhas. Além disso, é necessário soma térmica mínima para desencadear o processo germinativo de espécies como BRAPL (Carollo et al., 1997).

Os efeitos inibitórios sobre plantas daninhas foram mais pronunciados em alguns genótipos de sorgo. Como se trabalhou com quantidades de palha equivalentes entre os genótipos, essas diferenças podem ser atribuídas a variações qualitativas e quantitativas na liberação de substâncias pela parte aérea das plantas em decomposição. Resíduos de sorgo em decomposição liberam quantidades expressivas de substâncias hidrofilicas, em sua maioria ácidos fenólicos, como ferúlico, vanílico, p-hidroxibenzóico, p-cumárico e siríngico (Guenzi \& McCalla, 1966; Nicollier et al., 1983; Weston et al., 1999). As concentrações totais de substâncias fenólicas em plantas de sorgo variam entre genótipos, com a parte da planta e com a estação de crescimento (Ben-Hammouda et al., 1995).

Os resultados indicam que a produção diferencial de extratos radiculares hidrofóbicos em laboratório por genótipos de sorgo não se relaciona à supressão da densidade de plantas e do crescimento de Brachiaria plantaginea e Sida rhombifolia pela cobertura morta a campo; mostram também que o incremento da cobertura do solo com palha de sorgo reduz linearmente as infestações de B. plantaginea, $S$. rhombifolia e total de plantas daninhas. Além disso, conclui-se que a presença de resíduos da parte aérea de sorgo e milheto é mais importante na supressão de plantas daninhas do que a presença de resíduos das raízes dessas culturas.

\section{LITERATURA CITADA}

ALMEIDA, F. S. A alelopatia e as plantas. Londrina: Fundação IAPAR, 1988. 60 p. (IAPAR, Circular 53).

BEN-HAMMOUDA, M. et al. A chemical basis for differential allelopathic potencial of sorghum hybrids on wheat. J. Chem. Ecol., v. 21, n. 6, p. 775-786, 1995.

CAROLLO, C. M. et al. Germinação de leiteira (Euphorbia heterophylla) e papuã (Brachiaria plantaginea) submetidos a diferentes tratamentos de temperatura. In: SALÃO DE INICIAÇÃO CIENTÍFICA, 9., 1997, Porto Alegre. Resumos... Porto Alegre: UFRGS/PROPESQ, 1997. p. 99.

EINHELLIG, F. A. et al. Effects of root exudate sorgoleone on photosynthesis. J. Chem. Ecol., v. 19, n. 2, p. 369-375, 1993.

EINHELLIG, F. A.; SOUZA, I. F. Allelopathic activity of sorgoleone. J. Chem. Ecol., v. 18, n. 1, p. 1-11, 1992.

EINHELliG, F. A.; RASMUSSEN, J. A. Prior cropping with grain sorghum inhibits weeds. J. Chem. Ecol., v. 15, n. 3, p. 951-960, 1989.

FACELLI, J. M.; PICKETT, S. T. A. Plant litter: light interception and effects of an old-field plant community. Ecology, v. 72, n. 3, p. 1024-1031, 1991.

Planta Daninha, Viçosa-MG, v.22, n.1, p.1-10, 2004 
GONZALEZ, V. M. et al. Inhibition of a photosystem II eletron transfer reaction by the natural product sorgoleone. J. Agric. Food Chem., v. 45, p. 1415-1421, 1997.

GUENZI, W. D.; McCALLA, T. M. Phenolic acids in oats, wheat, sorghum, and corn residues and their phytotoxicity. Agron. J., v. 58, n. 3, p. 303-304, 1966.

HESS, D. E.; EJETA, G.; BUTLER, L. G. Selecting sorghum genotypes expressing a quantitative biosynthetic trait that confers resistance to Striga. Phytochemistry, v. 31, n. 2, p. 493-497, 1992.

HOFFMAN, M. L. et al. Separating the effects of sorghum (Sorghum bicolor) and rye (Secale cereale) root and shoot residues on weed development. Weed Sci., v. 44, n. 2, p. 402-407, 1996.

NETZLY, D. H. et al. Germination stimulant of witchweed (Striga asiatica) from hydrophobic root exudate of sorghum (Sorghum bicolor). Weed Sci., v. 36, n. 4, p. 441-446, 1988.

NICOLLIER, J. F.; POPE, D. F.; THOMPSON, A. C. Biological activity of dhurrin and other compounds from johnsongrass (Sorghum halepense). J. Agric. Food Chem. v. 31, n. 4, p. 744-748, 1983.

NIMBAL, C. I. et al. Phytotoxicity and distribution of sorgoleone in grain sorghum germplasm. J. Agric. Food Chem., v. 44, n. 5, p. 1343-1347, 1996.

PEIXOTO, M. F.; SOUZA, I. F.; SIQUEIRA, J. L. Resíduos culturais de sorgo e doses de imazamox no controle de Galinsoga parviflora e Richardia brasiliensis na cultura da soja em plantio direto. In: CONGRESSO BRASILEIRO DA CIÊNCIA DAS PLANTAS DANINHAS, 22., Foz do Iguaçu, 2000. Resumos... Londrina: Sociedade Brasileira da Ciência das Plantas Daninhas, 2000. p. 94.

PUTNAM, A. R.; DEFRANK, J.; BARNES, J. P. Exploration of allelopathy for weed control in annual and perennial cropping systems. J. Chem. Ecol., v.9, n. 8, p. 1001-1010, 1983.
RADOSEVICH, S.; HOLT, J.; GHERSA, C. Weed ecology: implications for management. 2.ed. New York: Wiley, 1997. p. 163-214.

RASMUSSEN, J. A. et al. Sorgoleone from root exudate inhibits mitochondrial functions. J. Chem. Ecol., v. 18, n. 1, p. 197-207, 1992.

RODRIGUES, J. C. et al. Determinação do conteúdo de sorgoleona nos exsudatos radiculares de híbridos de sorgo. R. Ceres, v. 48, n. 275, p. 49-54, 2001.

STRECK, E. V.; KAMPF, N.; KLAMT, E. Atualização da classificação taxonômica das unidades de mapeamento do levantamento e reconhecimento dos solos do Estado do Rio Grande do Sul. Porto Alegre: EMATER, 1999. 5 p. (Série Solos, v. 16, n. 9)

TEASDALE, J. R.; MOHLER, C. L. Light transmittance, soil temperature, and soil moisture under residue of hairy vetch and rye. Agron. J., v. 85, n. 3, p. 673-680, 1993.

THEISEN, G.; VIDAL, R. A.; FLECK, N. G. Redução da infestação de Brachiaria plantaginea em soja pela cobertura do solo com palha de aveia-preta. Pesq. Agropec. Bras., v. 35 , n. 4 , p. $753-756,2000$.

TREZZI, M. M. Avaliação do potencial alelopático de genótipos de sorgo. 2002. $127 \mathrm{f}$. Tese (Doutorado em Fitotecnia) - Universidade Federal do Rio Grande do Sul, Porto Alegre, 2002.

VIDAL, R. A. Amount of crop residues in no-till farming affects weed-crop ecosystems. 1995. $161 \mathrm{f}$. Thesis (Ph.D.) - Purdue University, West Laffayete, 1995.

VIDAL, R. A.; BAUMAN, T. T. Surface wheat (Triticum aestivum) residues, giant foxtail (Setaria faberi), and soybean (Glycine max) yield. Weed Sci., v. 44, n. 5, p.939943, 1996

WESTON, L. A.; NIMBAL, C. I.; JEANDET, P. Allelopathic potential of grain sorghum [Sorghum bicolor (L.) Moench] and related species. In: INDERJIT, DAKSHINI, K. M. M.; FOY, C. L. (Eds.) Principles and practices in plant ecology: allelochemical interactions. Boca Raton: CRC Press, 1999. p. 467-478. 